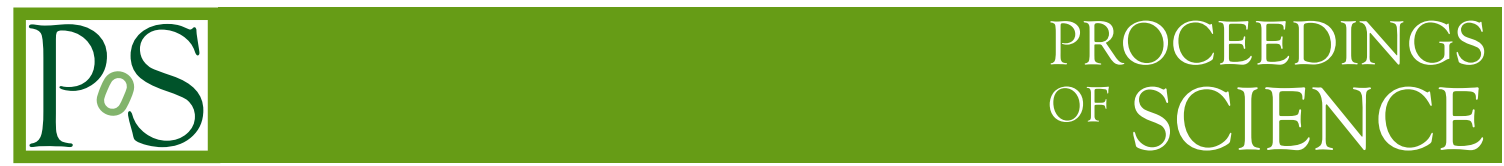

\title{
Drell-Yan Measurements with the CMS experiment
}

\author{
Ilya Gorbunov on behalf of the CMS Collaboration ${ }^{a, *}$ \\ ${ }^{a}$ International Intergovernmental Organization Joint Institute for Nuclear Research, \\ 6 Joliot-Curie St, Dubna, Moscow Region, Russia, 141980 \\ E-mail: Ilya.Gorbunov@cern.ch
}

In this note, the results of differential and inclusive Drell-Yan cross section measurements are summarized, including measurements of Forward-Backward asymmetry and Angular Coefficients. The measurements include both di-electron and di-muon decay channels.

40th International Conference on High Energy physics - ICHEP2020

July 28 - August 6, 2020

Prague, Czech Republic (virtual meeting)

\footnotetext{
${ }^{*}$ Speaker
} 


\section{Introduction}

The Drell-Yan (DY) process [1] considers lepton pairs production in pp-collisions and describes it by the s-channel exchange of $\gamma^{\star}$ and $Z$ bosons as illustrated in the Feynman diagram in Fig. 1 (left). For this process theoretical calculations are well established and available up to NNLO order in QCD. DY process measurements are important for a number of reasons: they allow tests of Standard Model (SM) predictions, they can provide constraints on parton distribution functions (PDFs), can be used to extract parameters of interest (for example $\sin ^{2} \theta_{W}$ ), can be an important source of background for many Beyond the Standard Model searches, for example it is an important source of background for many $Z^{\prime}$ searches.
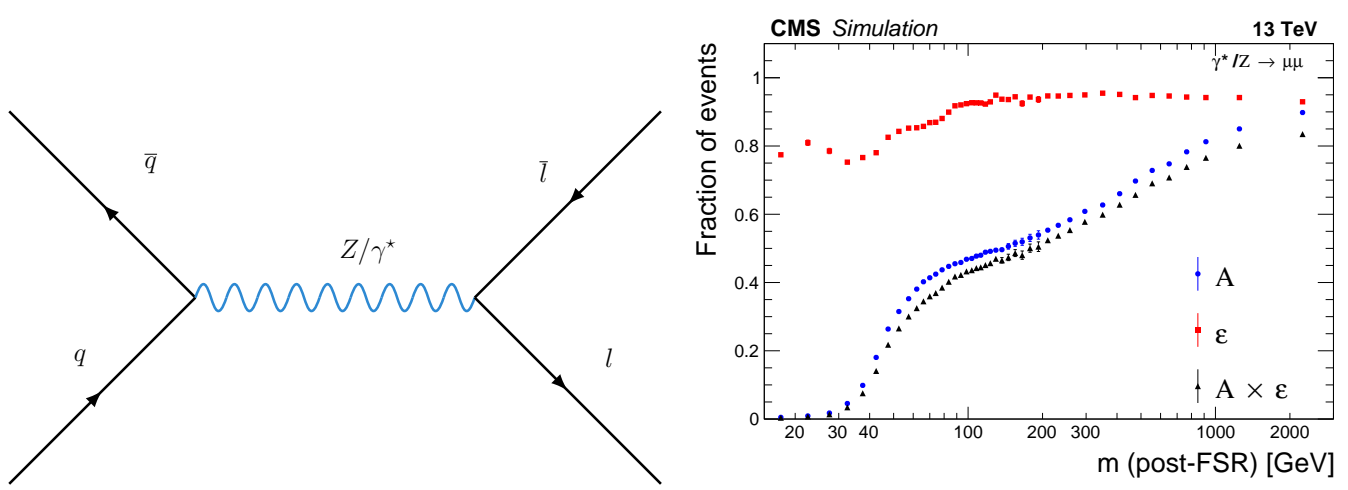

Figure 1: (left) The Drell-Yan lepton pair production. (right) Dimuon acceptance - blue circles, dimuon reconstruction, identification and isolation efficiencies - red squares, and Acceptance x Efficiency - black triangles [3].

Another motivation for the precision DY process measurements is to test different Monte Carlo models, evaluate production mechanism dynamics by studying parameters such as the Drell-Yan Angular Coefficients. By studying DY precess one can also probe non-perturbative QCD effects. The CMS experiment [2] has a large program of DY process studies.

\section{Drell-Yan Differential Cross Section Measurement at $\sqrt{s}=13 \mathrm{TeV}$}

The CMS Collaboration published $\sqrt{s}=13 \mathrm{TeV}$ results of differential Drell-Yan cross section measurements [3], using data corresponding to an integrated luminosity of $2.8(2.3) \mathrm{fb}^{-1}$ in the dimuon (dielectron) channel. Along with the cross section, the dimuon and dielectron acceptance, reconstruction, identification and isolation efficiencies were studied as a function of dilepton invariant mass, as shown in Fig. 1 (right). The acceptance effect is significant at low and decreases at high invariant mass ranges, almost reaching unity at masses above $2 \mathrm{TeV}$.

The total and fiducial cross section measurements are presented as a function of dilepton invariant mass in the range 15 to $3000 \mathrm{GeV}$, and compared with predictions at different orders in QCD and EW. The measurement were compared to different MC models, as shown in Fig. 2 (left) for the dimuon channel.

Fig. 2 (right) shows the differential DY cross section measured for the combination of the two channels. The measured differential cross sections are in good agreement with the theoretical 

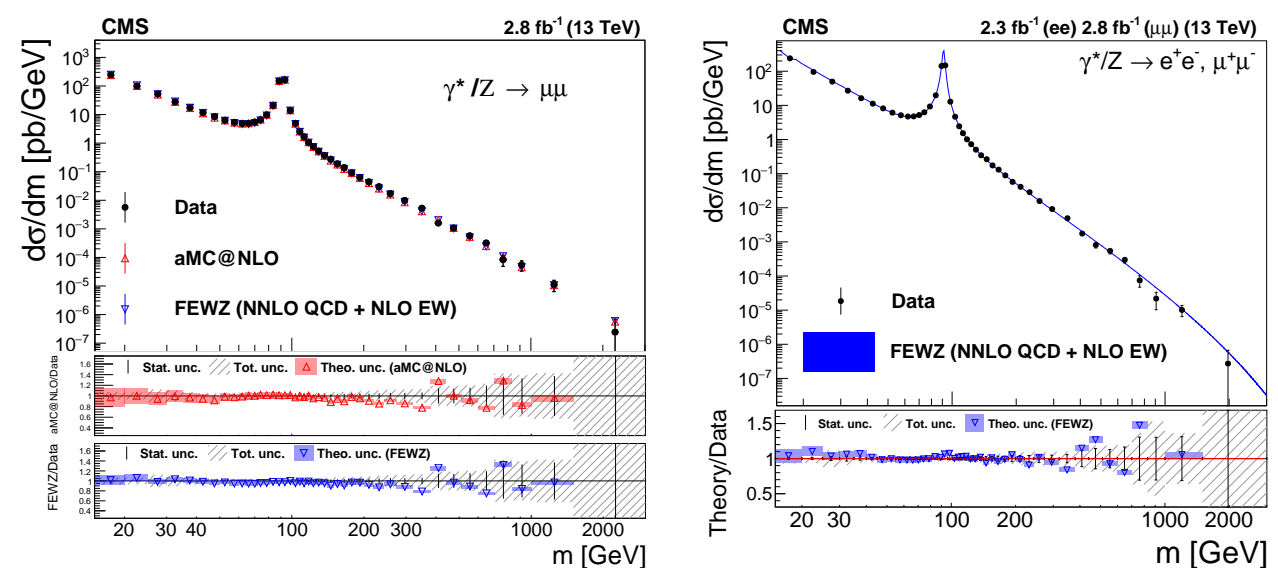

Figure 2: (left) Drell-Yan differential cross section vs. dimuon invariant mass. The cross section has been compared to MadGraph5_amc@NLO and FEWZ predictions [3]. (right) The Drell-Yan differential invariant mass cross section for combined (dielectron plus dimuon) channel compared to FEWZ predictions [3].
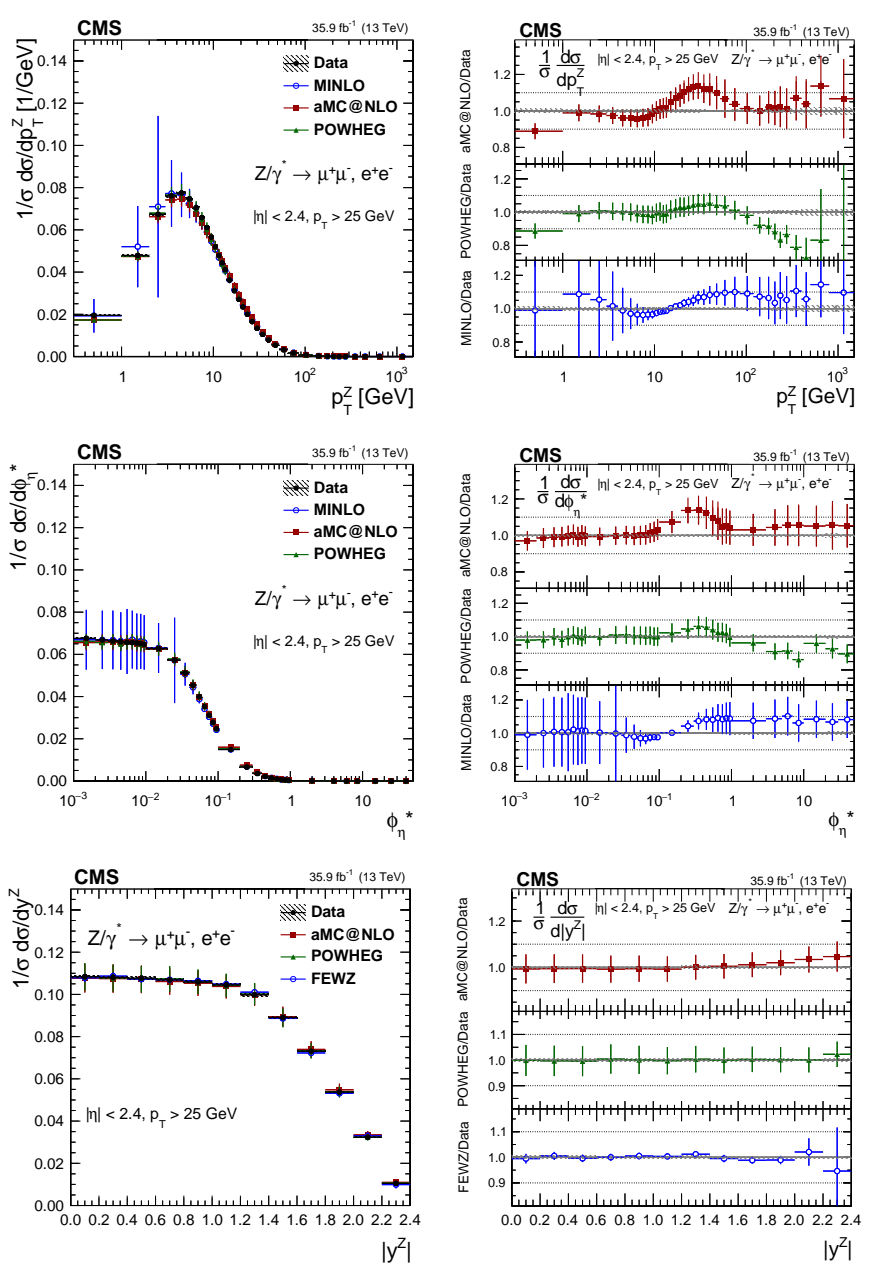

Figure 3: Differential cross sections of the transverse momentum $p_{T}$, the optimized angular variable $\phi_{\eta}^{\star}$, and the rapidity of lepton pairs [4]. 
calculations. The measurements are corrected to take into account effects of FSR. Fig. 2 (left and right) are in full phase space, not fiducial.

\section{3. $\mathrm{Z}$ production cross section at $13 \mathrm{TeV}$}

CMS also carried out measurements of differential cross sections of $\mathrm{Z}$ bosons produced in proton-proton collisions at $\sqrt{s}=13 \mathrm{TeV}$ decaying to dimuons and dielectrons [4]. Both absolute and normalised cross sections are measured. The data analysed were collected in 2016 with the CMS detector and correspond to an integrated luminosity of $35.9 \mathrm{fb}^{-1}$.

Table 1: $\mathrm{Z}$ boson inclusive cross section results in the dimuon and dielectron final states as well as the combination of the two [4].

\begin{tabular}{lllrllll}
\hline Cross section & \multicolumn{10}{c}{$\sigma \mathcal{B}[\mathrm{pb}]$} \\
$\sigma_{\mathrm{Z} \rightarrow \mu \mu}$ & 694 & \pm & 6 & (syst) & \pm & 17 & (lumi) \\
$\sigma_{\mathrm{Z} \rightarrow \mathrm{ee}}$ & 712 & \pm & 10 & (syst) & \pm & 18 & (lumi) \\
$\sigma_{\mathrm{Z} \rightarrow \ell \ell}$ & 699 & \pm & 5 & (syst) & \pm & 17 & (lumi) \\
\hline
\end{tabular}

The measured inclusive production cross sections shown in Tab. 1 agree with next-to-nextto-leading order quantum chromodynamics and branching fraction agree with next-to-next-toleading order quantum chromodynamics calculations. Differential cross sections of the transverse momentum $p_{T}$, the optimized angular variable $\phi_{\eta}^{\star}$, and the rapidity of lepton pairs are measured as shown in Fig. 3.
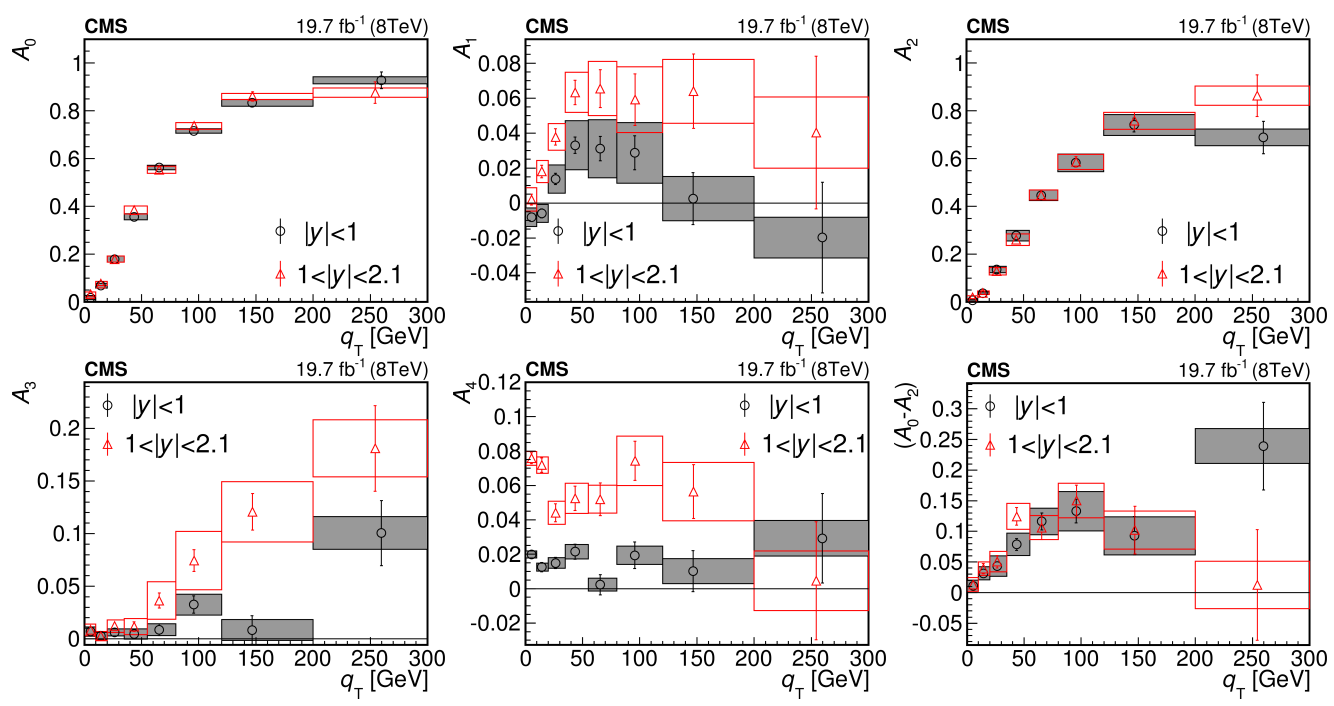

Figure 4: Comparison of the $A_{i}$ and $A_{0}-A_{2}$ coefficients measured in the Collins-Soper frame in bins of transverse momentum $q_{T}$ between $|y|<1$ (circles) and $1<q_{T}<2.1$ (triangles). The vertical bars represent the statistical uncertainties and the boxes the systematic uncertainties of the measurement [5].

The measurements are presented after correcting for detector effects and compared to theoretical predictions using fixed order calculations in QCD at different orders, including several approaches to include non-perturbative effects: parton shower approach, resummation as well as TMD approaches. The uncertainties of the measured normalised cross sections are smaller than $0.5 \%$ for $\phi_{\eta}^{\star}<0.5$ and for $p_{T}^{Z}<50 \mathrm{GeV}$. 


\section{Angular Coefficients}

Measurements of the five most significant angular coefficients, $A_{0}$ through $A_{4}$, for $\mathrm{Z}$ bosons produced in pp collisions at $\sqrt{s}=8 \mathrm{TeV}$ and decaying to muon pairs were carried out by the CMS Collaboration as a function of the transverse momentum and rapidity of the $\mathrm{Z}$ boson [5].

The integrated luminosity of the dataset collected with the CMS detector corresponds to 19.7 $\mathrm{fb}^{-1}$. These measurements provide comprehensive information about the $\mathrm{Z}$ boson production mechanisms, and are compared to the QCD predictions at leading order, next-to-leading order, and next-to-next-to-leading order in perturbation theory.

Fig. 4 shows the comparison of $A_{i}$ and $A_{0}-A_{2}$ measured in the Collins-Soper frame in bins of $q_{T}$ between $|y|<1$ (circles) and $1<y<2.1$ (triangles). The Lam-Tung relation was found to be violated at high transverse momentum and a significant rapidity dependence of the coefficients was observed.

\section{The Drell-Yan Forward-Backward Asymmetry Measurement}
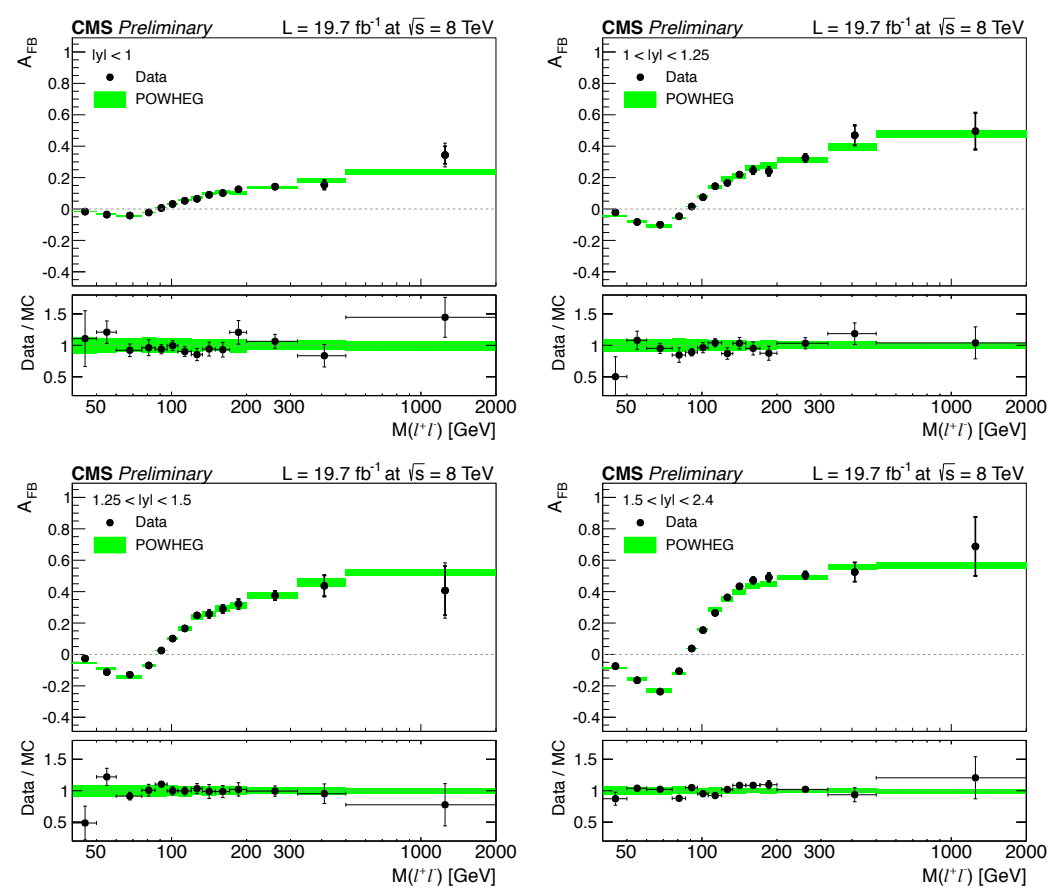

Figure 5: The combined $\left(\mu^{+} \mu^{-}\right.$and $\left.e^{-} e^{+}\right)$unfolded $A_{F B}$ distributions. The statistical (thick vertical bar) and statistical plus systematic (thin vertical bar) uncertainties are presented. The measurements are compared with the prediction of POWHEG. The total uncertainties (considering the statistical, PDF, and scale uncertainties) in the POWHEG prediction are shown as shaded bands [6].

A measurement of the forward-backward asymmetry $A_{F B}$ of oppositely charged lepton pairs ( $\mu \mu$ and ee) produced via $Z / \gamma^{\star}$ boson exchange in pp collisions at $\sqrt{s}=8 \mathrm{TeV}$ has been carried out by the CMS Collaboration [6]. The data sample corresponds to an integrated luminosity of 19.7 $\mathrm{fb}^{-1}$. The measurement has been done in bins of rapidity and dilepton invariant mass as shown in Fig. 5. 
The measurement of $A_{F B}$ is performed for dilepton masses between $40 \mathrm{GeV}$ and $2 \mathrm{TeV}$ and for dilepton rapidity up to 2.4, also extending to 5 in dielectron channel only (using electrons measured in a tracker free region of the CMS detector including forward hadron calorimeter). Measurements were corrected to take into account several effects including QED FSR, misalignment, reconstruction efficiency. No deviation from SM predictions is observed.

Using $A_{F B}$ results the CMS Collaboration published one of the most precise measurements of the $\sin ^{2} \theta_{W}$ [7]. Also, it has been shown that at the High Luminosity LHC era it will be possible to carry out the most precise measurement of the $\sin ^{2} \theta_{W}[8]$.

\section{Conclusion}

CMS has a huge program of high precision measurements, covering various SM processes in many different final states and at different centre of mass energies. High precision measurements of the Drell-Yan process (including weak mixing angle) are presented. Some of these measurements have been carried out at $13 \mathrm{TeV}$ ( $Z$ cross sections, DY differential invariant mass cross section). The measurements, as summarised in this note provide stringent tests of the SM predictions, some of which are also used to constrain parton distribution functions. Also, it has been shown that at the High Luminosity LHC era CMS will be able to improve the precision of its measurements.

\section{References}

[1] S.D. Drell and T.M. Yan, Massive Lepton-Pair Production in Hadron-Hadron Collisions at High Energies, Phys. Rev. Lett. 251970 316-320

[2] Chatrchyan, S. and others, The CMS experiment at the CERN LHC, JINST 32008 S08004

[3] CMS Collaboration, Measurement of the differential Drell-Yan cross section in proton-proton collisions at $\sqrt{s}=13 \mathrm{TeV}$, JHEP 12 (2019) 059, [hep-ex/1812 . 10529]

[4] CMS Collaboration, Measurements of differential Z boson production cross sections in protonproton collisions at $\sqrt{s}=13 \mathrm{TeV}$, JHEP 12 (2019) 061, [hep-ex/1812 . 10529]

[5] CMS Collaboration, Angular coefficients of Z bosons produced in pp collisions at $\sqrt{s}=8 \mathrm{TeV}$ and decaying to $\mu^{+} \mu^{-}$as a function of transverse momentum and rapidity, Phys. Lett. $B \mathbf{7 5 0}$ (2015) 154, [hep-ex/1504.03512]

[6] CMS Collaboration, Forward-backward asymmetry of Drell-Yan lepton pairs in pp collisions at $\sqrt{s}=8 \mathrm{TeV}$, Eur. Phys. J. C 76 (2016) 325, [hep-ex/1601.04768]

[7] CMS Collaboration, Measurement of the weak mixing angle using the forward-backward asymmetry of Drell-Yan events in pp collisions at 8 TeV, Eur. Phys. J. C 78 (2018) 701, [hep-ex/arXiv: 1806.00863]

[8] CMS Collaboration, A proposal for the measurement of the weak mixing angle at the HL-LHC, CMS-PAS-FTR-17-001, http://cds.cern.ch/record/2294888 